\title{
Energy exchanges during indentation tests in fresh-water ice
}

\author{
DEVINDER S. SODHI \\ U.S. Army Cold Regions Research and Engineering Laboratory, \\ 72 Lyme Road, Hanover, NH 03755, U.S.A.
}

\begin{abstract}
The data from a small-scale experimental study on ice-structure interaction are used to compute the energy exchanges that take place during creep deformation and intermittent and continuous crushing of ice. The energy supplied by the carriage is partly stored in the structural spring, partly converted to kinetic energy, partly dissipated in deforming and extruding the ice and partly dissipated as heat in the damping mechanisms of the structure. Except for the heat dissipation, all other forms of energy were computed from the experimental data, and the heat dissipation was computed from the energy balance using the first law of thermodynamics. Plots of all forms of energy are shown in graphical form, in which their relative magnitudes, times of occurrence and interplay can be seen. The main result of this study is the thesis that intermittent crushing or ice-induced vibration takes place whenever there is an imbalance between the rates of work done by the carriage and the indentor and that there are no vibrations when these rates of work are equal.
\end{abstract}

\section{INTRODUGTION}

Crushing is a common mode of ice failure during interaction between a moving ice sheet and a structure. Under certain conditions, the interaction leads to structural vibrations. Peyton (1968) and Blenkarn (1970) reported on ice-induced vibration of steel structures in Cook Inlet, Alaska. Recently, Jefferies and Wright (1988) reported on the vibration of Molikpaq, a $110 \mathrm{~m}$ wide structure, as a result of interaction with a multi-year seaice floe. Many small-scale experimental studies have been conducted to increase understanding of the manner in which such an interaction takes place. For extended discussions on this subject, the reader is referred to review papers by Sodhi (1988) and, for a slightly different point of view, Määttänen (1988).

In a recent paper, Sodhi (in press) reported on indentation tests that were conducted in a laboratory test basin by pushing vertical, flat indentors into the edges of floating, fresh-water ice sheets to study dynamic icestructure interaction during crushing failure of ice. The experimental data of that study are used here to compute the energy exchanges taking place between the structure and the ice. A brief description of the experimental set-up and the main results of the study are discussed first, followed by the results of the present analysis of the data.

\section{EXPERIMENTAL SET-UP}

The structure used in the study consisted of a mass and a spring (Fig. la) to simulate a single degree-of-freedom system. Two plates were attached to each other with four hinged links and two brackets with a spring between them. The top plate was mounted under the main carriage that spans the test basin. On the bottom plate were mounted the supports for the indentor plate and about $400 \mathrm{~kg}$ mass of lead weights. The spring consisted of two aluminum bars attached to each other by a number of rods. By removing or installing the rods in the middle of the spring, it was possible to change the unsupported span of the aluminum bars, thus changing the stiffness of the spring.

Indentor plates of different widths were supported on three load cells so that the true interaction force could be measured, as opposed to interpretation of the structural response as that force. Besides measurement of the interaction forces, the displacements of the carriage and of the indentor were measured separately with respect to a fixed datum. Further, the relative displacement of the indentor with respect to the carriage was measured independently. Hereafter, the relative displacement will be designated as structure displacement because it is equal to the deformation of the spring. An accelerometer was also placed on the lower plate to measure its acceleration during the interaction. These measurements provided a complete set of data on the interaction forces and the response of the structure.

All experiments were done with fresh-water, columnar ice, which was grown in the basin after seeding with a fine mist of water sprayed in the air. Ice sheets of different thicknesses $(15-57 \mathrm{~mm})$ were grown. For a particular value of spring stiffness, experiments were done by moving 

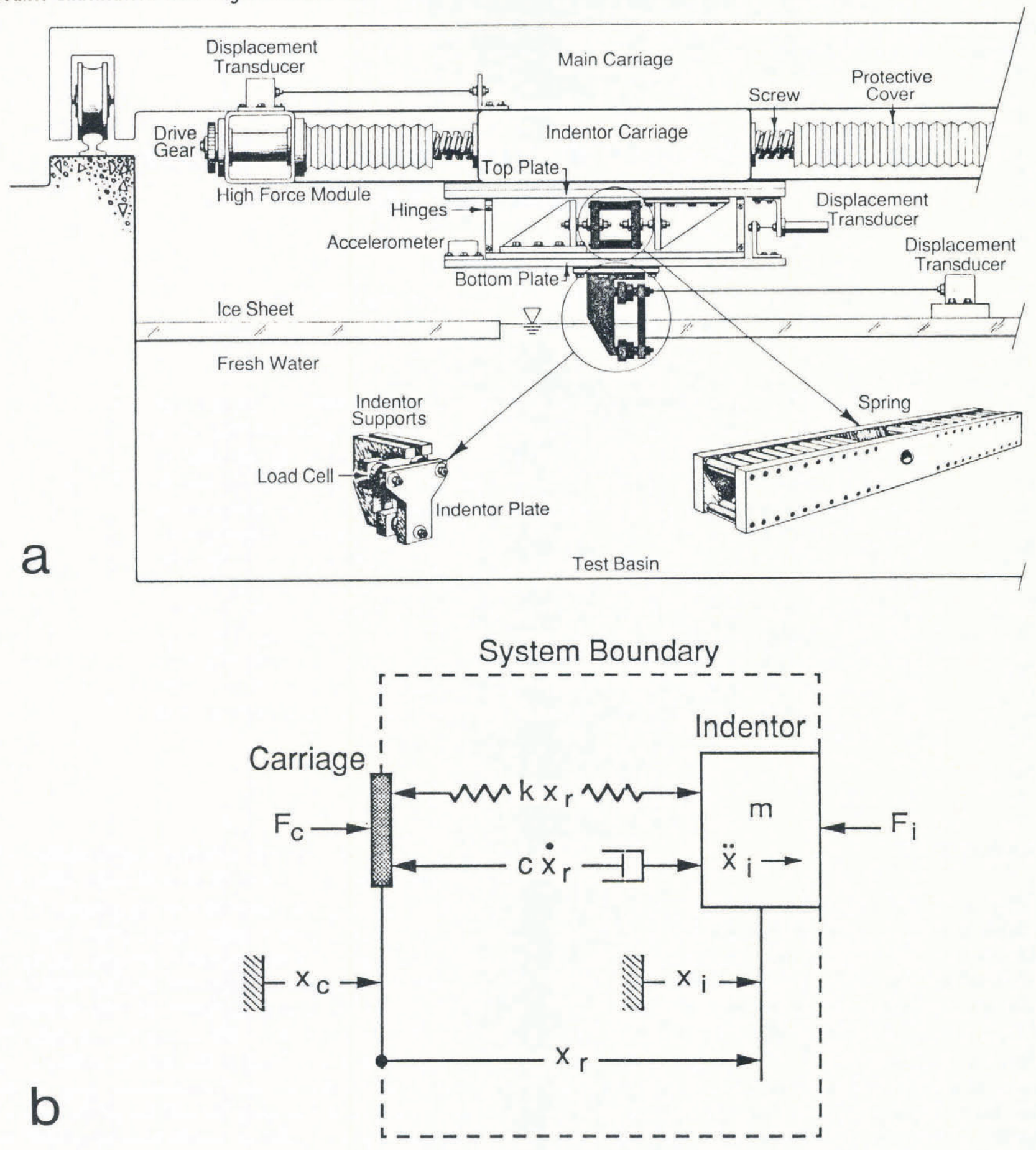

Fig. 1. Schematic sketch of the apparatus: (a) experimental set-up, (b) system and its elements. The explanation for the different symbols is given in the text.

the carriage at three different velocities. For tests conducted at high velocities, the carriage velocity was changed in discrete steps without stopping an experiment.

During the course of the experiments, the following parameters were varied: ice thickness, indentor width, carriage velocity and spring stiffness. Further details on these tests are discussed by Sodhi (in press).

\section{RESULTS}

Three modes of ice behaviour were observed during the study: creep deformation at low indentation velocities, intermittent crushing at intermediate indentation velo- cities and continuous crushing at high indentation velocities. At times, the structure vibrated at its natural frequency, a situation referred to as resonance.

Typical records of the experimental data for the four different interactions are shown in Figures 2-6. Figure 4 shows part of the data in Figure 3 at an expanded time scale. Each figure presents time records of interaction force $\left(F_{\mathrm{i}}\right)$, displacement of the carriage $\left(x_{\mathrm{c}}\right)$ and the indentor $\left(x_{\mathrm{i}}\right)$, and structure displacement $\left(x_{\mathrm{r}}\right)$ of the indentor relative to the carriage. It can be seen that the compatibility condition of $x_{\mathrm{r}}=x_{\mathrm{c}}-x_{\mathrm{i}}$ is satisfied, within the error bounds of the displacement tranducers, at all times.

The force records can be interpreted in terms of 
Test No. 64
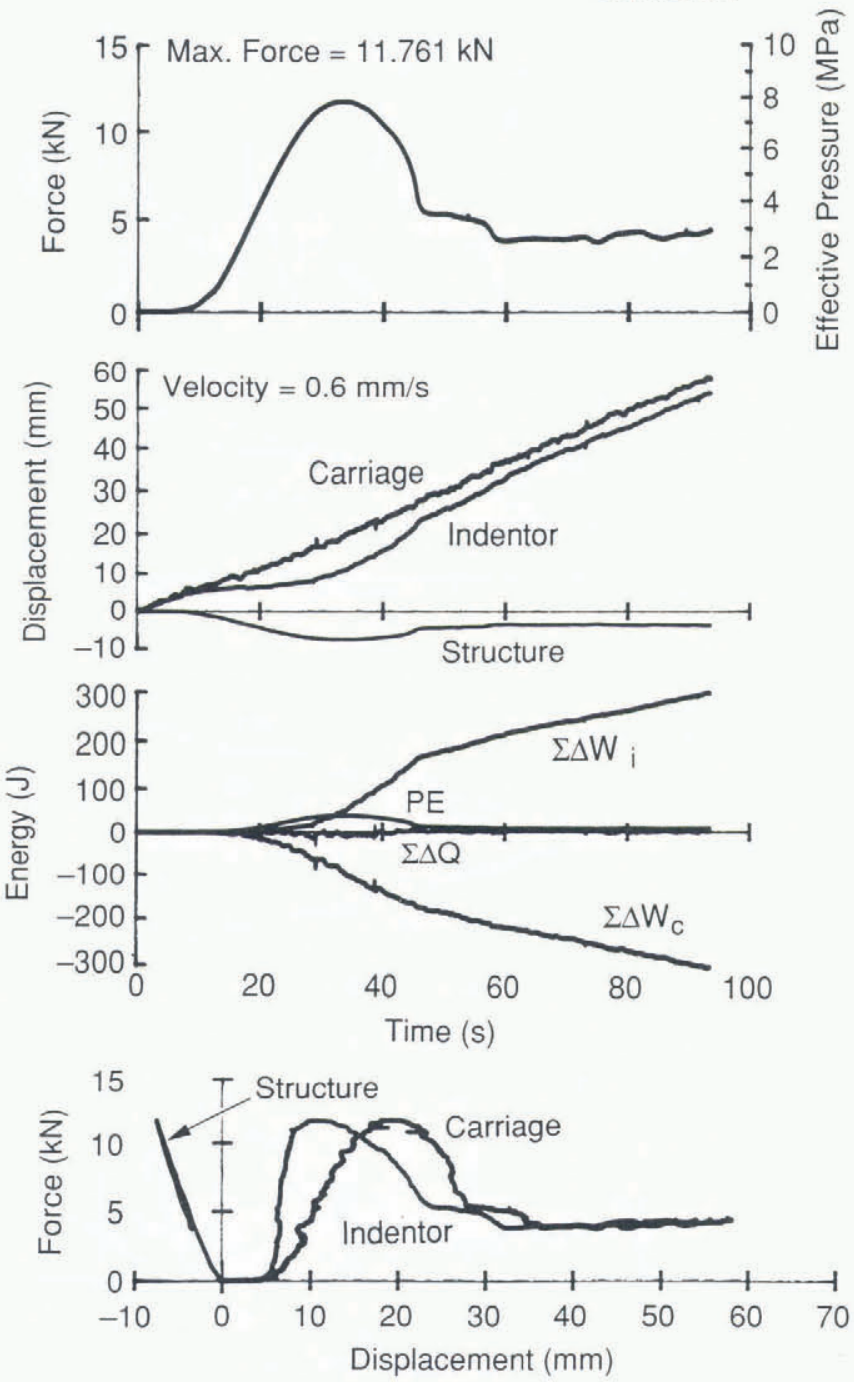

Fig. 2. Results of a test in which creep deformation of ice took place.

effective pressure by dividing the force scale by the contact area, and the scale on the righthand side reflects the pressure scale for a particular test. The maximum effective pressure was in the range $8-13 \mathrm{MPa}$ during the creep deformation and intermittent crushing modes, whereas it was very low, between 1 and $2 \mathrm{MPa}$, during the continuous crushing mode.

To obtain force-displacement graphs, the corresponding values of force and displacement are paired for each scan of the data. On the bottom of each figure, three forcedisplacement plots are shown for the carriage, the indentor and the structure. The force values in these three plots at a given instant are assumed to be the same because the carriage, the indentor and the structural spring experience more or less the same force, as discussed later. The areas under these three plots represent the cumulative energy transferred or stored; these are also shown in Figures 2-6 with respect to time. The computational procedure for obtaining these plots is discussed below.

The apparatus (Fig. lb) is considered as a thermodynamic system. Any heat transferred to the system is considered positive and any work done by the system is considered positive. During a process between two states,
Test No. 204
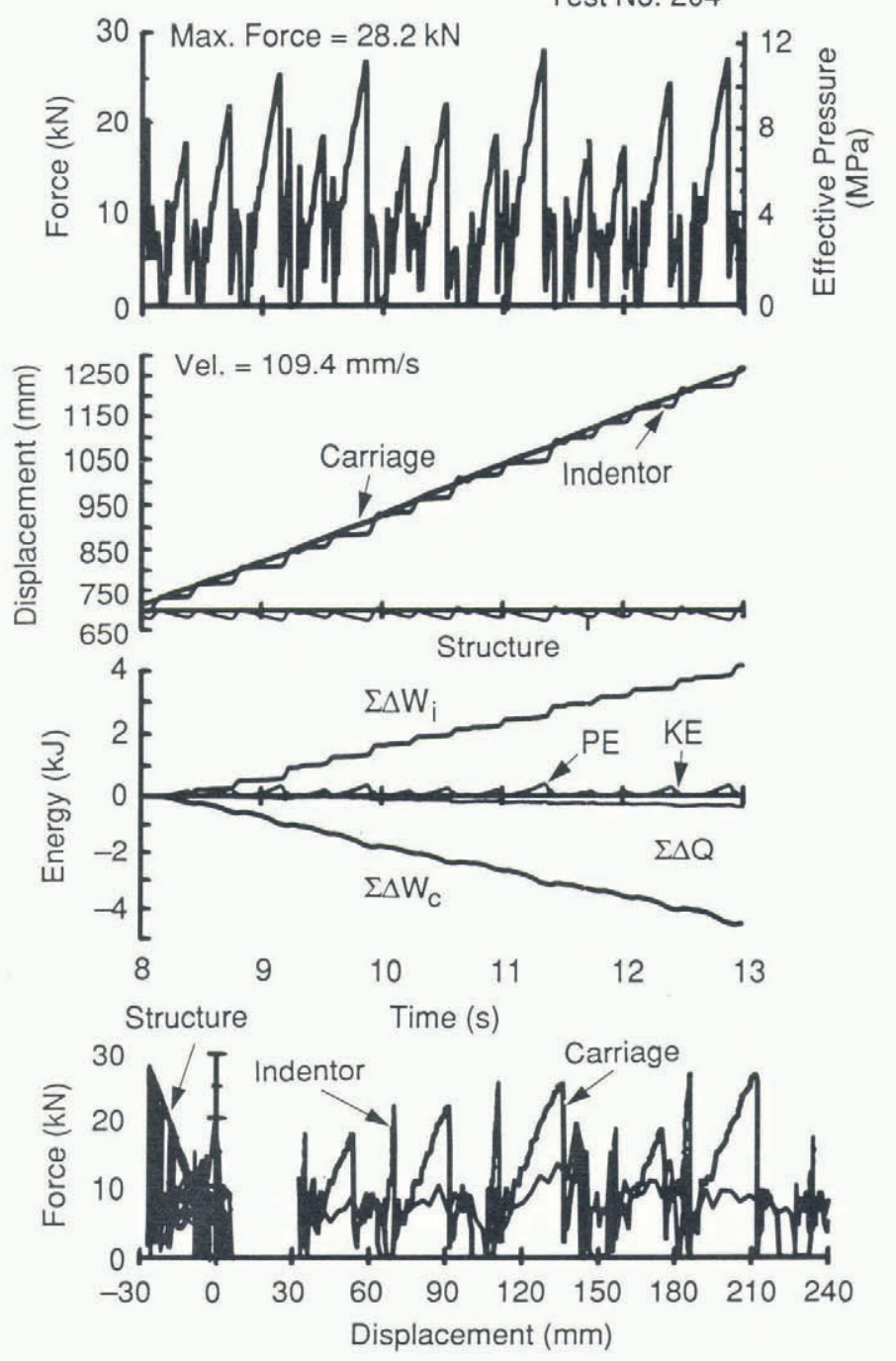

Fig. 3. Results of a test in which ice-structure interaction resulted in intermittent crushing. In the plots of displacement time-histories, the relative displacement of the structure with respect to the carriage has been denoted as structure displacement and plotted with respect to the origin in each part of the figure. Similarly, in the force-displacement plots, displacements of the carriage and the indentor have been translated to the origin shown in the bottom part of the figure.

the energy exchange satisfies the following equation according to the first law of thermodynamics

$$
\Delta Q=\Delta(P E)+\Delta(K E)-\Delta W_{\mathrm{c}}+\Delta W_{\mathrm{i}},
$$

where $\Delta Q$ is the incremental heat transferred to the system, $\Delta(P E)$ the incremental change in its potential energy, $\Delta(K E)$ the incremental change in kinetic energy of the system, and $\Delta W_{\mathrm{c}}$ and $\Delta W_{\mathrm{i}}$ the incremental work done by the carriage and the indentor, respectively. The computation of $\Delta W_{\mathrm{c}}$ and $\Delta W_{\mathrm{i}}$ between any two instants of time can be accomplished by multiplying the average force by the corresponding incremental displacements of the carriage and the indentor, respectively. The incremental change in kinetic energy is computed from $\Delta\left(0.5 m \dot{x}^{2}{ }_{\mathrm{i}}\right)$, where $m$ is the mass of the vibrating system, and $\dot{x}_{\mathrm{i}}$ the indentor velocity obtained by differentiating the displacement record. The incremental potential energy stored in the structure is given by $\Delta\left(0.5 K x^{2}{ }_{\mathrm{r}}\right)$ where $K$ is 

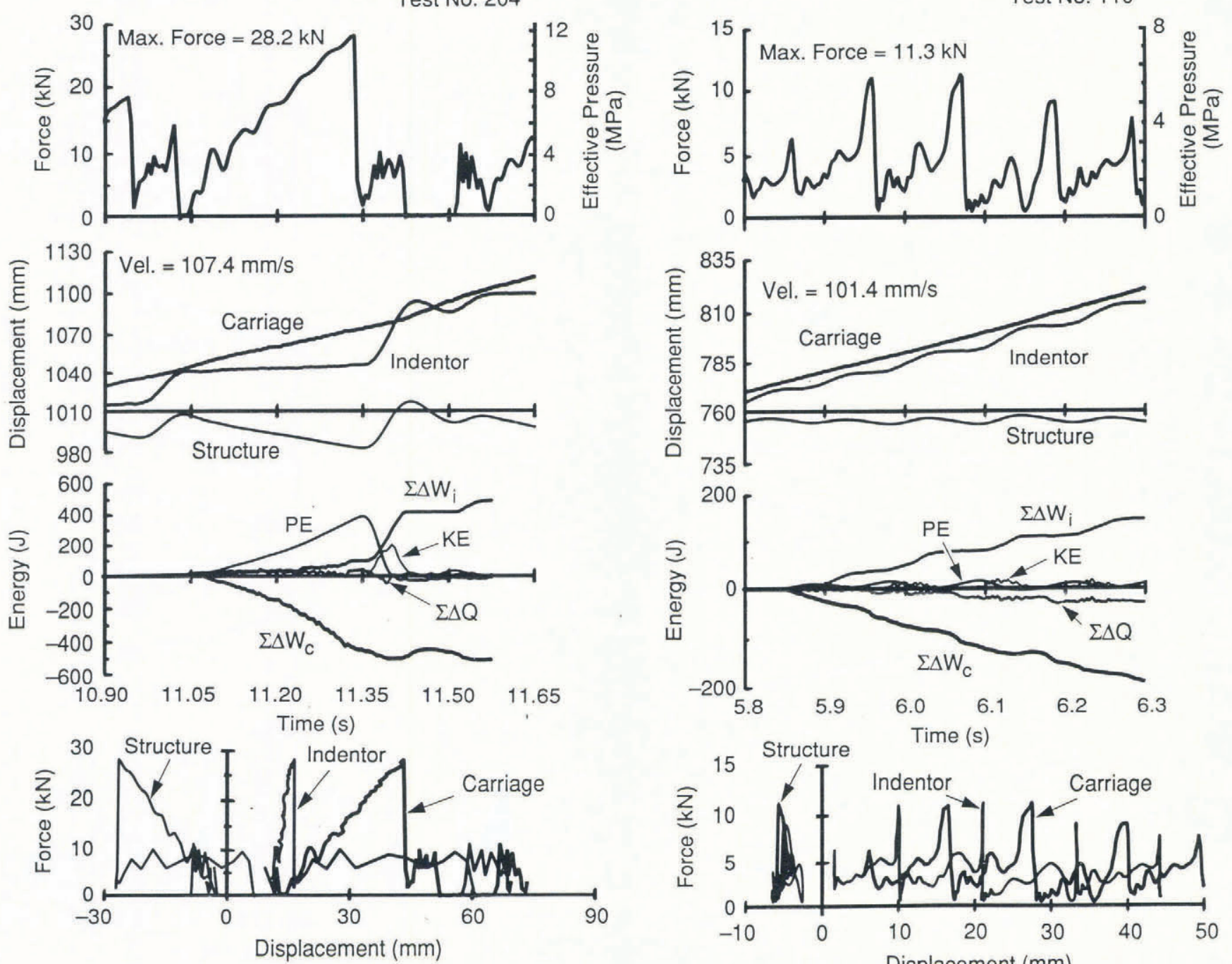

Fig. 4. Detailed plot of results shown in Figure 3. In the plots of displacement time-histories, the relative displacement of the structure with respect to the carriage has been denoted as structure displacement and plotted with respect to the origin of the figure. Similarly, in the force-displacement plots, displacements of the carriage and the indentor have been translated to the origin shown in the bottom part of the figure.

the structural stiffness. The stiffness was determined from the slope of the graph between the interaction force and the structural displacement, during the phase when the interaction force increased and the vibrations were minimal.

When there is no vibration taking place during a process, we have $\Delta(P E)-\Delta W_{\mathrm{c}}+\Delta W_{\mathrm{i}}=0$, which means that the incremental increase in the potential energy of the system is equal to the incremental work done on the system, or vice versa. This equation can be rewritten for a conservative system as shown below:

$$
K x_{\mathrm{r}} \Delta x_{\mathrm{r}}-F_{\mathrm{c}} \Delta x_{\mathrm{c}}+F_{\mathrm{i}} \Delta x_{\mathrm{i}}=0,
$$

where $F_{\mathrm{C}}$ and $F_{\mathrm{i}}$, respectively, are the forces exerted by the carriage and the indentor. During continuous crushing and steady-state creep deformation of ice, the structure

displacement $x_{\mathrm{r}}$ is constant (i.e. $\Delta x_{\mathrm{r}}=0$ ), and we get $F_{\mathrm{c}}$ $=F_{\mathrm{i}}$ because the rates of the carriage and indentor displacement are equal. During the initial stages of creep deformation (e.g. Fig. 2 for $t<45 \mathrm{~s}$ ), we get $F_{\mathrm{c}}=K x_{\mathrm{r}}$ and $F_{\mathrm{c}}=F_{\mathrm{i}}$ by using the compatibility condition (i.e. $\Delta x_{\mathrm{c}}=$ $\left.\Delta x_{\mathrm{i}}+\Delta x_{\mathrm{r}}\right)$ and the condition that two of three displacements are independent of each other.

During a process when there are structural vibrations taking place, Equation 2 does not hold, and $\Delta Q$ can be computed from Equation 1 as given below:

$$
\Delta Q=K x_{\mathrm{r}} \Delta x_{\mathrm{r}}+\Delta\left(0.5 m \dot{x}_{\mathrm{i}}^{2}\right)-F_{\mathrm{c}} \Delta \mathrm{x}_{\mathrm{c}}+F_{\mathrm{i}} \Delta x_{\mathrm{i}} .
$$

Equation 3 gives an estimate of the energy dissipation in the frictional and damping mechanisms of the structure. In Equation 3, all the variables were measured during the experiments except the force, $F_{c}$, exerted by the carriage. 
Test No. 206
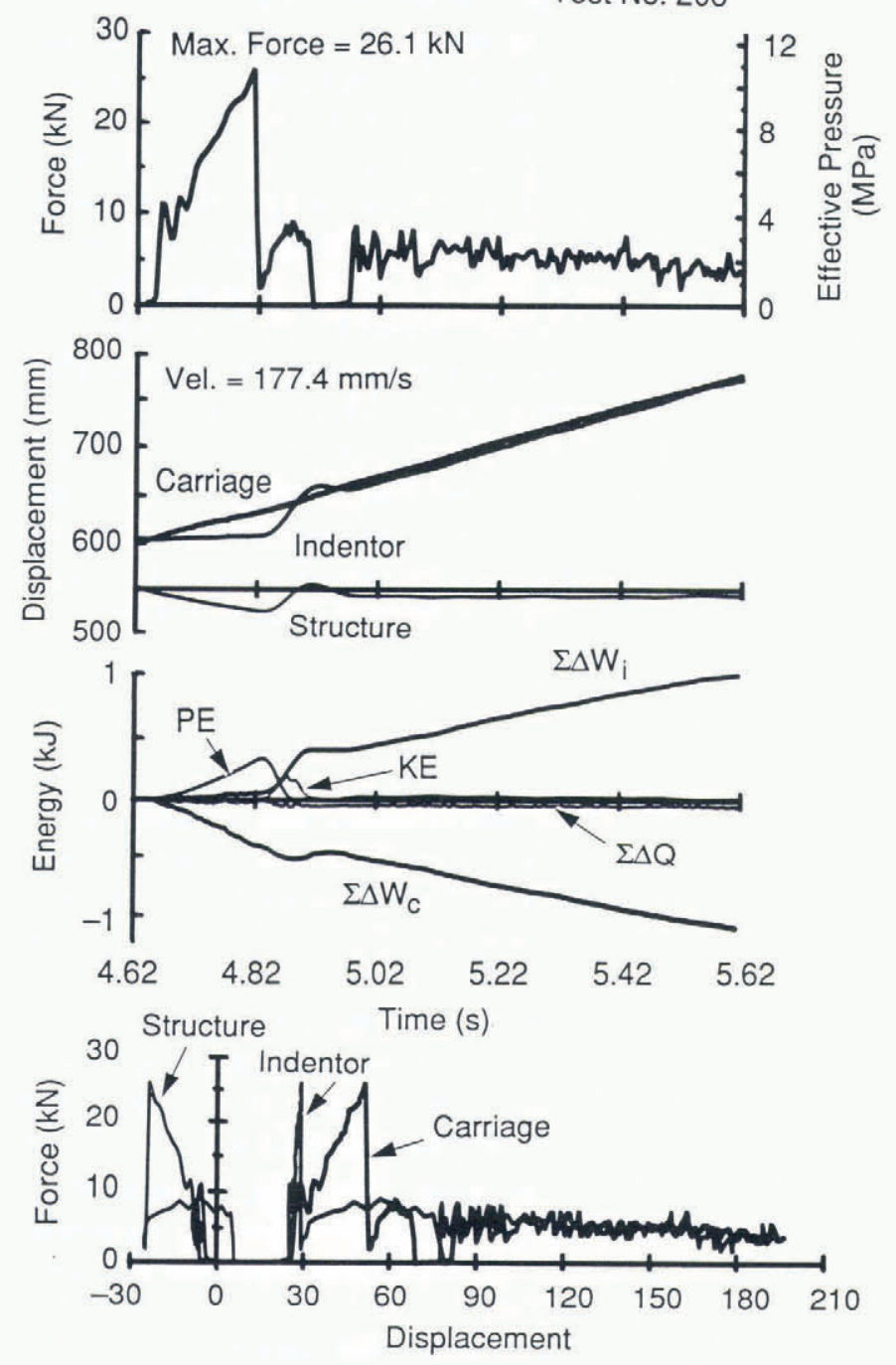

Fig. 6. Results of a test which continuous ice crushing took place. In the plots of displacement time-histories, the relative displacement of the structure with respect to the carriage has been denoted as structure displacement and plotted with respect to the origin of the figure. Similarly, in the force-displacement plots, displacements of the carriage and the indentor have been translated to the origin shown in the bottom part of the figure.

Invoking the equilibrium of the indentor and the carriage, according to the free-body diagrams shown in Figure 1b, we get $F_{\mathrm{c}}=F_{\mathrm{i}}+m \ddot{x}_{\mathrm{i}}$. By substituting $F_{\mathrm{c}}$ in Equation 3, $\Delta Q$ was computed using the acceleration of the indentor measured during the experiments.

Plots of the cumulative work done by the carriage $\left(\Sigma \Delta W_{\mathrm{c}}\right)$ and the indentor $\left(\Sigma \Delta W_{\mathrm{i}}\right)$, the potential energy stored in the structural spring $(P E)$, the kinetic energy $(K E)$ of the system and the energy dissipated in the form of heat $(\Sigma \Delta Q)$ are shown in Figures 2-6.

\section{DISCUSSION}

In the plots of cumulative energy shown in Figures 2-6, it can be seen that most of the energy supplied by the carriage is dissipated by the indentor into the deformation, breakage and extrusion of ice in front of the indentor. Different types of ice-structure interaction and energy exchanges occur at different indentation velocities.
It is useful to introduce a concept of generalized impedance of the ice at the indentor interface as the ability of the ice to move at a velocity under the action of a force. In this way, we can look at the transmission of energy from the carriage to the ice through the structure. Whenever the velocity of the indentor is equal to that of the carriage (e.g. steady-state creep or continuous crushing), this situation is characterized as impedance matching because all the energy is transmitted without any change in the stored potential energy of the structure. When the indentor velocity is different from that of the carriage, all the energy is not transmitted from the carriage to the ice, and part of it is stored in the structure and released later when the indentor is able to move through the ice after ice failure. The situation described here is similar to that in testing machines (Sinha and Frederking, 1979), where the strain rate measured by an extensometer placed on an ice specimen is less than that calculated from the cross-head speed. The stiffness of a testing machine plays an important part in the rate of loading a specimen.

During creep deformation of ice at low indentation velocities $\left(<1 \mathrm{~mm} \mathrm{~s}^{-1}\right)$, the interaction force increased first and then decreased to a constant value (Fig. 2). In this case, the kinetic energy of the system was negligible due to small magnitudes of velocity. During the initial interaction with undamaged ice, the indentor lagged behind the carriage, caused extensive micro-cracking in the ice, and then attained a constant displacement rate behind the carriage. The potential energy was stored in the structure during the initial interaction period, and it was later released until a steady-state condition of constant energy dissipation by the indentor, equal to that supplied by the carriage, was reached.

During intermittent crushing at the intermediate indentation rate (Figs. 3-5), the interaction takes place in a cyclical manner, which is also discussed by Sodhi and Nakazawa (1990). In each cycle, the interaction force increases, causing a relative displacement between the carriage and the indentor and a storage of potential energy in the structure. When the force reaches a certain value, the ice fails, resulting in a sudden forward motion of the indentor. The stored structural energy is used to move the indentor forward and to extrude the crushed ice out of the path of the indentor. During this time, as shown by the plots of energy in Figure 4, there is an exchange of energy between the stored potential energy, the kinetic energy of the system and the work done by the indentor, whereas the work done by the carriage $\Delta W_{\mathrm{c}}$ during that time interval is small. Sometimes, the indentor travels backward. During these periods, the interaction force is zero and, hence, the work done by the indentor is also zero. A new cycle begins with increase of interaction force and storage of energy in the structure (as shown in Figs. 3 and 4).

From the plots of cumulative energy, the portions of energy transformed or exchanged into different forms can be established at any instant in time. For example, the energy plots in Figure 4 indicate that just before the ice failure, $78 \%$ and $22 \%$ of the energy supplied by the carriage is stored in the spring and dissipated by the indentor, respectively. At the end of an intermittent crushing event (Fig. 4), the portions of energy supplied by the carriage are $95 \%$ dissipated into the ice by the indentor and $5 \%$ dissipated in the damping mechanisms of the structures. 
When the structure vibrates at its natural frequency (Fig. 5), the increase and decrease in the potential energy and the kinetic energy of the structure approximately follow a sinusoidal form. The overall effect is that the major portion of the energy supplied by the carriage is dissipated in the ice. Lastly, the balance of energy $(\Sigma \Delta Q)$ is the heat dissipated in overcoming the frictional and damping force of the structure, and its plot increases with time when there are structural vibrations. From data given in Figure 5, the damping factor (defined as the ratio of equivalent viscous damping coefficient to the critical damping coefficient) can be calculated from the estimation of energy loss $(\Sigma \Delta Q)$ per cycle. The damping factor was found to be 0.21 , which is close to the value of 0.14 obtained from the ratio of any two consecutive amplitudes of the system when it was displaced from its static equilibrium position and released with zero initial velocity.

At high indentation velocities, the interaction switches suddenly from intermittent crushing to continuous crushing, as shown in Figure 6 . The stored potential energy in the structure remains constant, whereas the rate of energy supplied by the carriage equals that dissipated by the indentor. The surprising result of continuous crushing is the low effective pressure generated during the interaction. There is a need to investigate the reason for this low value of effective pressure.

Among the plots of energy shown in Figures 2-6, the rates of energy transfer or exchange are highly variable because of different rates and modes of ice failure.

Similar energy computations have been given by Nakazawa and Sodhi (1990), but these were done only during the period of increasing interaction forces. Jordaan and Timco (1988) estimated the different energy components during the interaction. Because the displacements of the structure and the indentor were not measured independently, they deduced the structure's displacement from its stiffness. They limited their analysis to one single failure event. Moreover they theorized that the increase in interaction force takes place during the extrusion process, which was not found to be the case in the observations made during the experimental study described above.

In Figures $2-6$, the cumulative work done by the indentor $\left(\Sigma \Delta w_{\mathrm{i}}\right)$ was found to be a non-decreasing function. This signifies that there is always energy dissipation into the ice and no transfer of energy from the ice to the structure. This rules out any possibility of ice-induced vibration as a result of negative damping, as proposed by Blenkarn (1970) and Määttänen (1988).

The above discussion pertains to the results of experiments done in this study, in which the ratio of the structural stiffness to the effective stiffness of ice was small ( 0.5 to 0.1 ). In the case of rigid structures, the stored potential energy in the ice sheet may be greater than that in the structure. New sets of experiments are needed to study this type of ice-structure interaction.

\section{GONCLUSION}

Energy exchanges during ice-structure interaction are computed using the data obtained from a small-scale experimental study. On the whole, most of the energy supplied by the carriage was dissipated by the indentor in deforming and/or extruding the ice, but the rate of energy input to the structural system does not always equal that of the output. Whenever the ice is not able to move out of the way of the indentor at the same rate as that of the carriage, there will be relative displacement of the indentor with respect to the carriage, resulting in storage of energy in the structure. The stored energy is later released after ice failure, when the ice is able to move under reduced levels of force, and exchange into kinetic energy and work done in deforming and extruding ice. This imbalance between the rates of work done by the carriage and the indentor results in ice-induced vibrations. During steady-state processes of continuous creep and crushing, the rate of the energy supplied by the carriage equals that dissipated by the indentor, resulting in no structural vibrations.

\section{REFERENCES}

Blenkarn, K.A. 1970. Measurement and analysis of ice forces on Cook Inlet structures. Second Annual Offshore Technology Conference, 1970 Proceedings. Houston, Texas. Vol. 2. Houston, TX, Offshore Technology Conference, 365378. (OTC 1261).

Jefferies, M.G. and W.H. Wright. 1988. Dynamic response of "Molikpaq" to ice-structure interaction. In Sodhi, D.S., C.H. Luk, and N.K. Sinha, eds. OMAE 1988 Houston. Proceedings of the Seventh International Conference on Offshore Mechanics and Arctic Engineering presented at ... Houston, Texas, February 7-12, 1988. Vol. 4. Arctic engineering and technology. New York, American Society of Mechanical Engineers, 201-220.

Jordaan, I.J. and G.W. Timco. 1988. Dynamics of the icecrushing process. J. Glaciol., 34(118), 318-326.

Määttänen, M. 1988. Ice-induced vibrations of structures - self-excitation. In Sacki, H. and K.-i. Hirayama, eds Proceedings, the 9th International Symposium on Ice, 23-27 August 1988, Sapporo, Japan. Vol. 2. Delft, International Association for Hydraulic Research. Committee on Ice Problems, 658-665.

Nakazawa, N. and D.S. Sodhi. 1990. Ice forces on flat, vertical indentors pushed through floating ice sheets. CRREL Spec. Rep. 90-14.

Peyton, H.R. 1968. Sea ice forces. Ice pressures against structures. Ottawa, National Research Council, 117-123. (Technical Memorandum 92.)

Sinha, N.K. and R.M.W. Frederking. 1979. Effect of test system stiffness on strength of ice. POAC 79. The Fifth International Conference on Port and Ocean Engineering Under Arctic Conditions at the Norwegian Institute of Technology, August 13-18, 1979. Proceedings. Vol. 1. Trondheim, University of Trondheim. Norwegian Institute of Technology, 708-717.

Sodhi, D.S. 1988. Ice-induced vibrations of structures. In Sacki, H. and K.-i. Hirayama, eds. Proceedings, the 9th International Symposium on Ice, 23-27 August 1988, Sapporo, Japan. Vol. 2. Delft, International Association for Hydraulic Research. Committee on Ice Problems, 625657.

Sodhi, D.S. In press. Ice-structure interaction during indentation tests. In Jones, S.J., R. McKenna, J. Tillotson, and I.J. Jordaan, eds. IUTAM/IAHR Sympo- 
sium on Ice-Structure Interaction, St. John's, Newfoundland, Canada, August, 14-17, 1989. Berlin, etc., Springer-Verlag. Sodhi, D.S. and N. Nakazawa. 1990. Frequency of intermittent ice crushing during indentation tests. IAHR 90. Proceedings of the 10th International Symposium on
Ice, August 20-23, 1990, Espoo, Finland. Vol. 3. Espoo, Helsinki University of Technology, 277-289.

The accuracy of references in the text and in this list is the responsibility of the author, to whom queries should be addressed. 